\title{
Influence of platform position on stray grain nucleation in Ni-based single-crystal dummy blade clusters
}

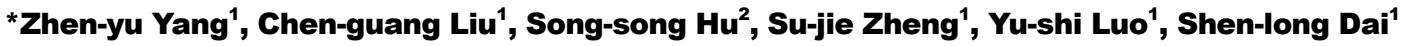 \\ 1. Science and Technology on Advanced High Temperature Structural Materials Laboratory, Beijing Institute of Aeronautical Materials, \\ Beijing 100095, China \\ 2. School of Materials Science and Engineering, Xiangtan University, Xiangtan 411105, Hunan, China
}

\begin{abstract}
Stray grains are the most severe of the solidification defects that occur in the industrial single-crystal blade preparation process. In this study, a single-crystal dummy blade cluster with different crystal orientations controlled by the seeding method was prepared, and the influence of the position of the circular platform (relative to the sample and furnace body) on stray grain nucleation was investigated. Results show that the microstructure of the circular platforms could be divided into the center, expansion, and stray grain regions. The inside of the circular platform facing the center of the cluster is more prone to stray grain formation than the outside of the circular platform facing the furnace body. With an increase in the distance between the circular platform and the bottom of the dummy blade cluster, the stray grain region expands, whereas the expansion region narrows. The stray grain is slightly aggravated with increase of the misorientation. Finally, the mechanism underlying the influence of platform position on the formation of stray grains in single-crystal dummy blade clusters is discussed based on the temperature evolution during directional solidification.
\end{abstract}

Key words: Ni-based single-crystal superalloy; directional solidification; stray grain; nucleation; dummy blade cluster

CLC numbers: TG146.1 5 Document code: A Article ID: 1672-6421(2021)05-442-08

\section{Introduction}

Ni-based single-crystal turbine blades, which are the core hot-section components of aircraft turbines and industrial gas turbines ${ }^{[1]}$, exhibit excellent hightemperature comprehensive performance attributable to two aspects ${ }^{[2]}$. First, the single-crystal blade contains no grain boundaries, which are the weak link in terms of mechanical properties at high temperatures. Second, the $<001>$ direction of a single-crystal blade corresponding to the smallest elastic modulus coincides with the maximum stress direction ${ }^{[3]}$. Therefore, during the preparation of single-crystal blades, the crystal orientation and stray grains should be strictly controlled.

Stray grains are the most severe solidification defects that occur in the industrial single-crystal blade preparation process ${ }^{[4-6]}$. Many research efforts have

\section{*Zhen-yu Yang}

Male, doctoral candidate. Research interests: microstructure analysis, hightemperature mechanical performance and casting process control of nickelbased single crystal superalloys and turbine blades.

E-mail: xuanshangyiyi@163.com

Received: 2021-03-21; Accepted: 2021-09-08 focused on the formation of stray grains ${ }^{[7-10]}$ and found that an abrupt variation in the cross-section, such as the platform, serves as the most likely position for stray grain formation. Meyer et al. ${ }^{[1]}$ illustrated that stray grains nucleated in thermal undercooling zones as a result of macroscopically curved isotherms sweeping through the platform extremities, which was confirmed by experiments and simulations ${ }^{[5,12,13]}$. Research results indicated that with increasing the withdrawal rate ${ }^{[14,15]}$, platform length ${ }^{[16]}$, and misorientation ${ }^{[6]}$, and with decreasing the critical nucleation undercooling ${ }^{[17]}$, the tendency toward the formation of stray grains at the platform increased. Sun et al. ${ }^{[18-20]}$ found that dendrite deformation can cause the formation of a large-angle boundary when a high-order branching dendrite grew into the platform region via loop growth, which was a kind of mechanism for the formation of stray grains. These findings indicate the direction for inhibiting the formation of stray grains at platform.

To improve the production efficiency of singlecrystal blades and reduce production costs, multi-blade and multi-layer clusters are often used for directional solidification investment casting with grain selection 
method or seeding method in industrial production. In a cluster, the platform positions relative to the furnace body and cluster bottom are different. Ma et al. ${ }^{[13]}$ studied the temperature evolution and defect formation in single-crystal blade clusters via experiments and simulations and found that asymmetric thermal conditions in the blade cluster during directional solidification allow stray grains to form inside the platform facing the center of the cluster. Since the grain selection technology is mainly used to study the formation of the stray grains at platform, the crystal direction of the single crystal casting is not precisely controlled, so the direct relationship between the formation of the stray grains and the position of the platform has not been established. In fact, Xuan et al. ${ }^{[6]}$ reported that the influence of crystal orientation on the formation of stray grains cannot be ignored.

In this study, the seeding method was used to precisely control the single-crystal orientation, and a dummy blade with three circular platforms was fabricated to investigate the influence of platform position on the nucleation of stray grains in multi-blade and multi-layer clusters.

\section{Experimental procedure}

The second-generation Ni-based single-crystal superalloy DD6 was used, and its nominal chemical composition is shown in Table 1. A dummy blade cluster, the structure and size of which are shown in Fig. 1, was used to fabricate a single-crystal sample using the seeding method. The wax dummy blades were formed by 3D printing (ProJet MJP 3600W, 3D Systems) and then assembled into a cluster, as shown in Fig. 1. The ceramic shell mold was fabricated by an investment casting procedure. Seeds with a size of $3.5 \mathrm{~mm} \times 3.5 \mathrm{~mm} \times 30 \mathrm{~mm}$ were cut from a single-crystal rod prefabricated by the grain selection method along the $<001>$ direction or deviated from the seed axial by $15^{\circ}$. The $<010>$ direction of the seed was set parallel or perpendicular to the side surface of the seed. The seeds were plugged into the mold with the $<010>$ direction facing the center of the cluster. The $<001>$ direction of seeds with misorientation of $15^{\circ}$ was converged to the center of the cluster. The assembly was fixed on a water-cooled copper chill plate in an industrial Bridgman directional furnace. During directional solidification, the furnace chamber was first evacuated to a partial pressure of approximately $0.56 \mathrm{~Pa}$. Then, the mold was heated to $1,520{ }^{\circ} \mathrm{C}$ and held for $3-5 \mathrm{~min}$. The liquid metal at $1,520^{\circ} \mathrm{C}$ was poured into the mold and held for another 3-5 min. The mold was then withdrawn at a rate of $50 \mu \mathrm{m} \cdot \mathrm{s}^{-1}$.

Following directional solidification, the mold was removed from the dummy blade cluster. The casting was macro-etched by a mixture of $\mathrm{H}_{2} \mathrm{O}_{2}$ and $\mathrm{HCl}$ to macroscopically inspect the solidification defects. The casting was then machined using wire electro discharge machining and polished for microstructural

Table 1: Nominal chemical composition of DD6 (wt.\%)

$\begin{array}{llllllllll}\text { Cr } & \text { Co } & \text { Mo } & \text { W } & \text { Ta } & \text { Re } & \text { Nb } & \text { Al } & \text { Hf } & \text { Ni } \\ 4.3 & 9 & 2 & 8 & 7.5 & 2 & 0.5 & 5.6 & 0.1 & \text { Bal. }\end{array}$

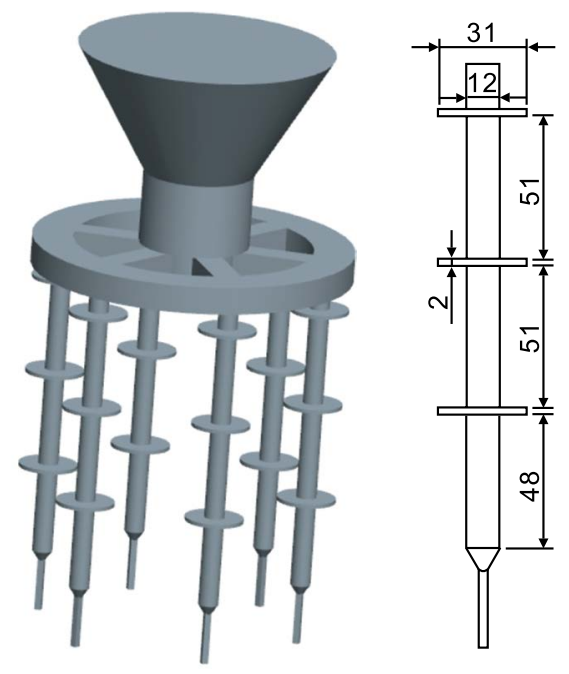

Fig. 1: Schematic diagram of dummy blade cluster structure (a) and size (b) (unit: $\mathrm{mm}$ )

inspection, followed by etching with a mixture of $14 \% \mathrm{HNO}_{3}$, $28 \% \mathrm{HF}$, and $58 \% \mathrm{C}_{3} \mathrm{H}_{8} \mathrm{O}_{3}$. An optical microscope (DM4000, Leica) was used to observe the microstructure. A scanning electron microscope (SEM; SUPRA 55, Zeiss) equipped with EBSD and EDS was used for crystal orientation and elemental distribution measurements.

\section{Results}

The solidification defects commonly form at the melt-back region during directional solidification with the seeding method. Figure 2 shows the microstructure of a meltback region for a seed with different crystal orientations. A coarse, cell-like microstructure was obtained below the meltback interface, and no randomly oriented broken dendrite was formed. This indicates that the current directional solidification process can ensure the stability of the meltback region before withdrawing the mold from the heat zone of a furnace. No stray grain is found above the melt-back interface at the seed perimeter for different seed crystallographic orientations. Therefore, the seeding method can be used for controlling the crystal orientation of dummy blades.

Upon removing the mold, it is found that one seed naturally departs from the dummy blade without any extra loading force, whereas the remaining seeds merge with the casting as a whole, as shown in Fig. 3. Macroscopic inspection shows that the orientation of all dummy blades is consistent with that of the seeds, and solidification defects form only on the circular platforms. This indicated that the liquid metal solidified epitaxially along the seeds during directional solidification. As shown in Fig. 3(b), the seed that naturally departs from the dummy blade is caused by the formation of an oxide film on the top of the seed during the heating stage. The liquid melt convection caused by the pouring process does not break the oxide film, resulting in isolation of the liquid melt between the seed and casting. Therefore, there is no markedly metallurgical combination between the seed and dummy blade. In other 

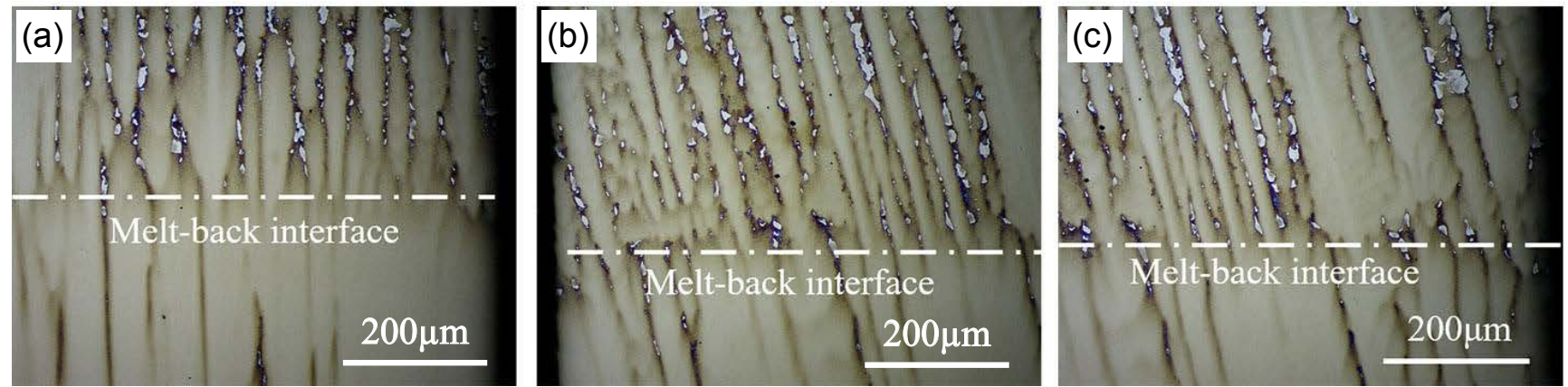

Fig. 2: Microstructure of melt-back region: (a) <001> seed; (b) converging side; and (c) diverging side with a misorientation of $15^{\circ}$

(a)

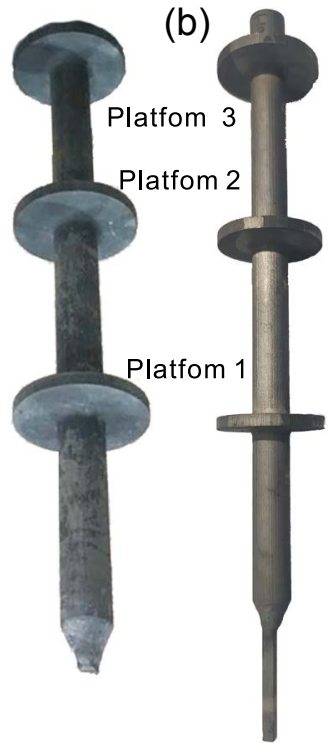

Fig. 3: Macrostructure of a dummy blade with its seed (a) and after detachment of its seed (b) experiments, it was also found that some solidification defects could be attributed to the formation of an oxide film on the top of the seed ${ }^{[21]}$. The influence of seed oxidation on the formation of solidification defects needs to be further investigated; however, since this is not the main research topic of the present study, no further explanation is given here.

The microstructure of the bottom surface of Platform 1 of a dummy blade with $<001>$ orientation is shown in Fig. 4 . The side of the platform immediately facing the furnace body was defined as the outside, whereas the side of the platform facing the center of the cluster was defined as the inside. During directional solidification, the $<001>$ direction of the single crystal is parallel to the dummy blade axis, and the $<100>$ direction squarely faces outside the platform. As shown in Fig. 4(a), the microstructure of the bottom surface of Platform 1 could be divided into three parts from the center to the edge: the center region, expansion region, and stray grain region. The microstructure and corresponding crystal orientation are shown in Fig. 4(b-e). The most common morphology of dendrite arm arrangement appears in the center (a)

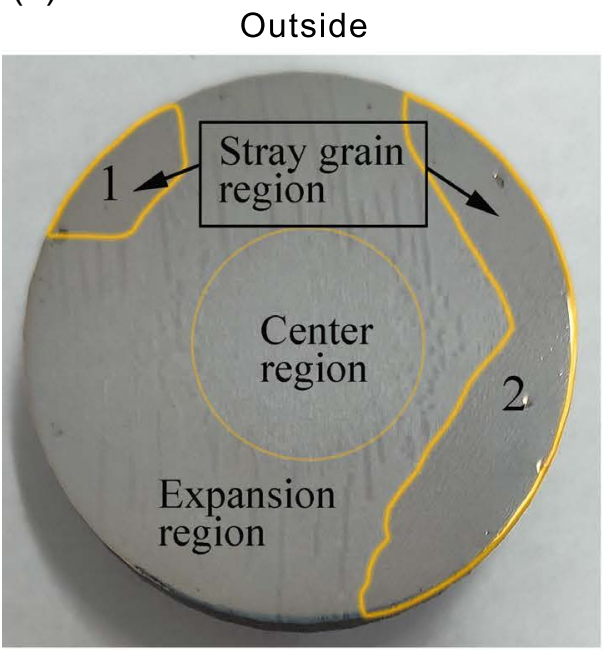

Inside
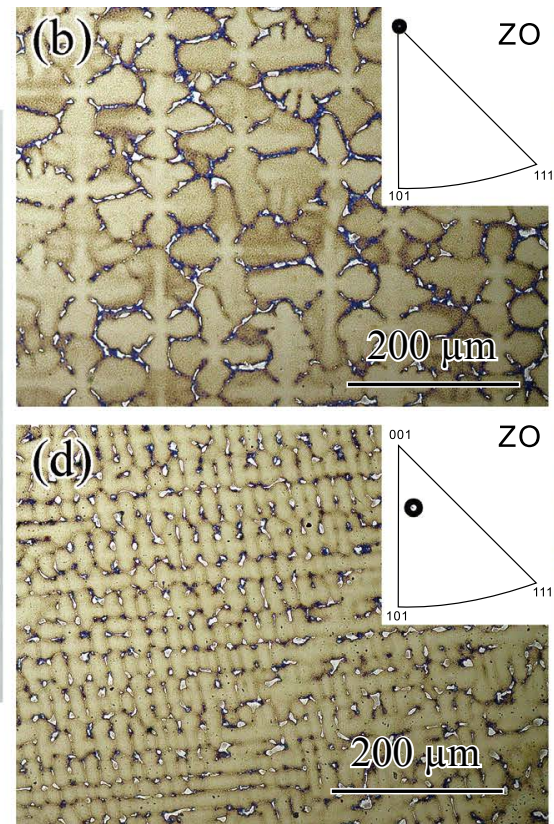
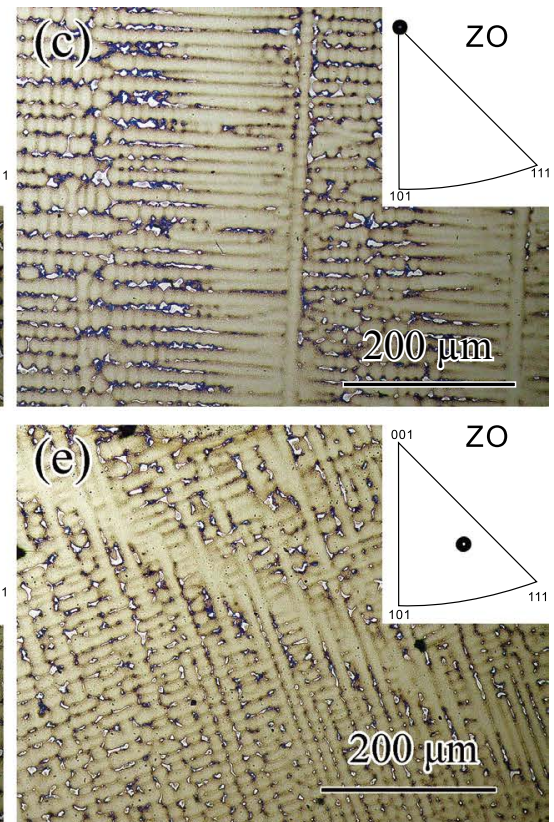

Fig. 4: Transverse macrostructure (a) and microstructures of Platform 1 of the dummy blade with <001> orientation in center region (b), expansion region (c), and stray grain region (d, e) (the insets in (b-e) are the corresponding EBSD inverse pole figures) 
region, represented by four-petal flower-like shapes. The crystal orientation in the expansion region is consistent with that in the center region. The dendrites are formed by expansion of the edge dendrites in the center region via high-order branching, with the characteristics of long secondary and tertiary branches, as shown in Fig. 4(c). As illustrated by the microstructure in the stray grain region shown in Fig. 4(d), the dendrites exhibit highorder branching growth. The main characteristic of the stray grain region is that the crystal orientation is different from that of the expansion and center regions, and there is an obvious grain boundary between the expansion region and stray grain region, as shown in Figs. 4(a and e). Notably, since the seeds are plugged into the mold with the $<010>$ direction facing the center of the cluster, the position of the stray grain region is consistent with the $<110>$ direction of the matrix grain. The matrix grain grows into this position by multiple high-order branching, causing it to lag behind the positions which are parallel or perpendicular to the $<010>$ direction of single crystal. This allows for the formation of a large undercooling for the nucleation and growth of stray grains.

The microstructures of Platforms 2 and 3 are shown in Fig. 5 and Fig. 6, respectively. Like Platform 1, Platforms 2 and 3 are also divided into center, expansion, and stray grain regions with similar microstructures, as shown in Fig. 5(b-e) and Fig. 6(b-e). With the increasing distance between the platform and the bottom of the cluster, the expansion area gradually (a)

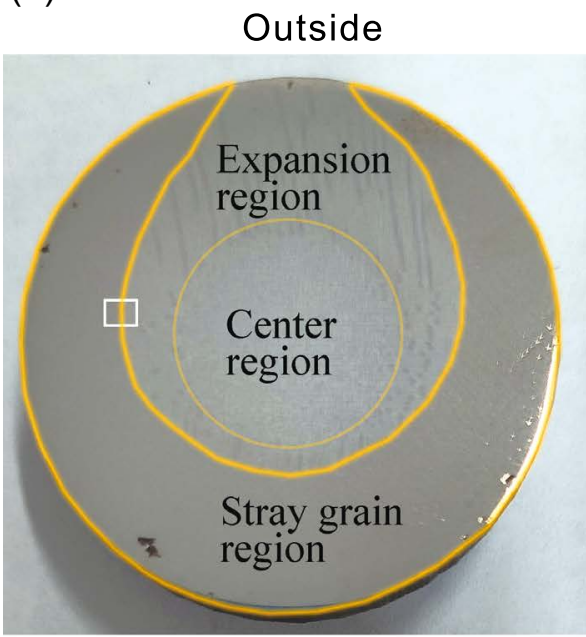

Inside
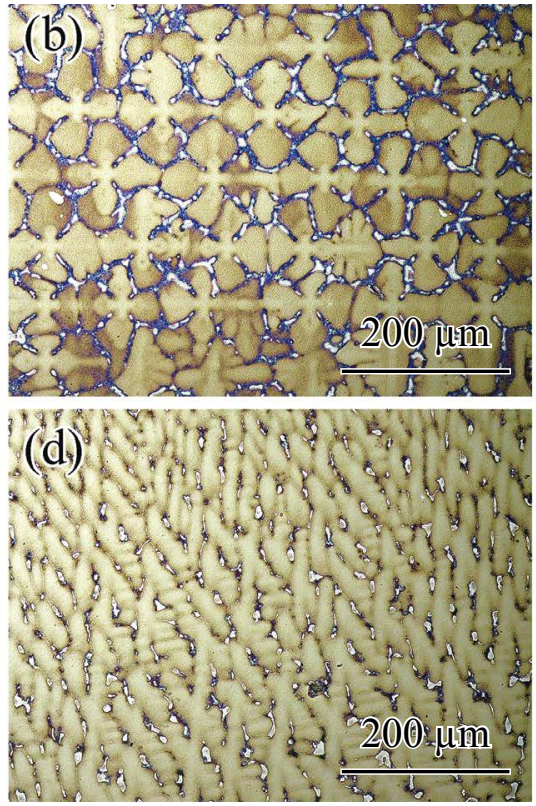
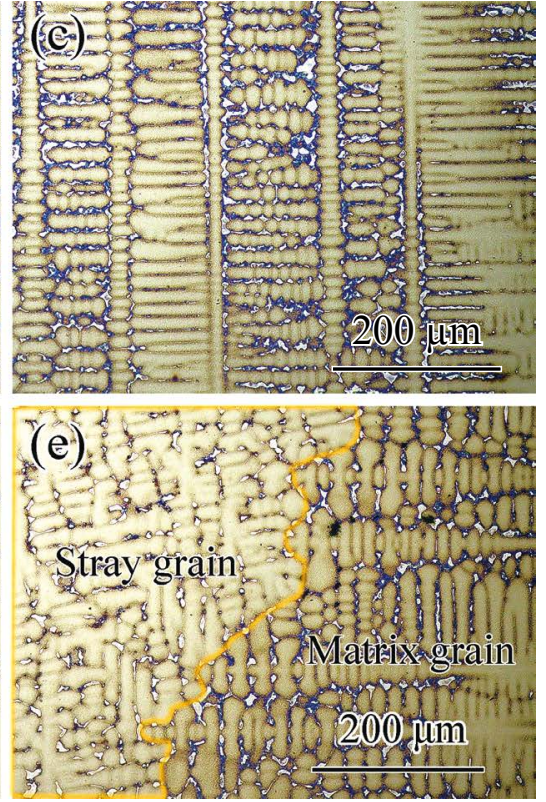

Fig. 5: Transverse macrostructure (a) and microstructures of Platform 2 of the dummy blade with <001> orientation in center region (b), expansion region (c), stray grain region (d), and grain boundary marked by the white square in (a) (e)

(a)

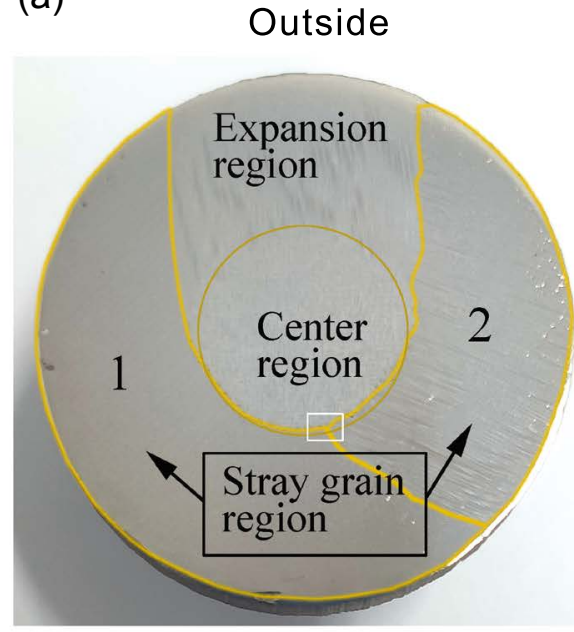

Inside
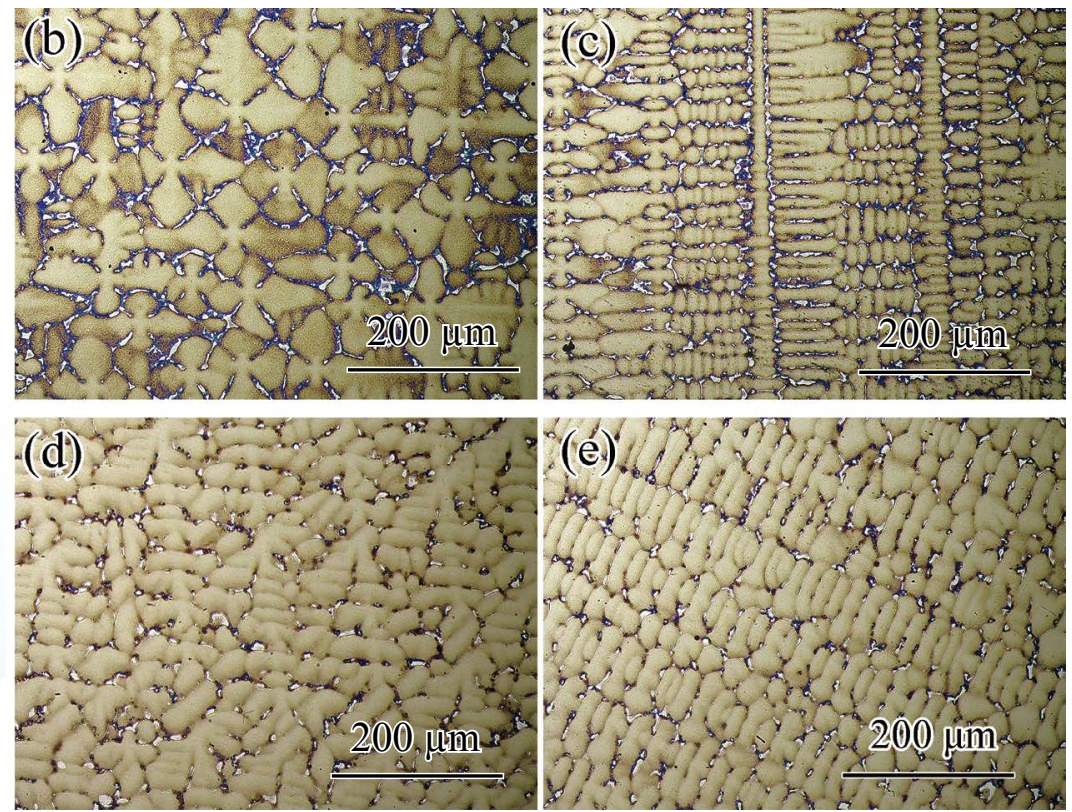

Fig. 6: Transverse macrostructure (a) and microstructures of Platform 3 of the dummy blade with <001> orientation in center region (b), expansion region (c), and stray grain region (d, e) 
decreases, while the stray grain area increases correspondingly. However, the center region basically remains unchanged, except for a slight reduction for Platform 3. At the same time, the stray grain region is in direct contact with the center region on Platform 3, as shown in Fig. 6(a). It should be noted that for all the three platforms, the outside (facing the furnace body) is occupied by the expansion region. This indicates that the probability of stray grain formation on the outside is lower than that at other positions.

Platform 3 shows features markedly different from Platforms 1 and 2. As shown in Fig. 7, obvious dark matter is observed at the grain boundary between the center and stray grain regions. To clarify the composition of the dark material, EDS analysis was performed on the zone marked by the white square in Fig. 7, and the results are shown in Fig. 8. The EDS results show that the dark matter does not contain any elements, indicating that

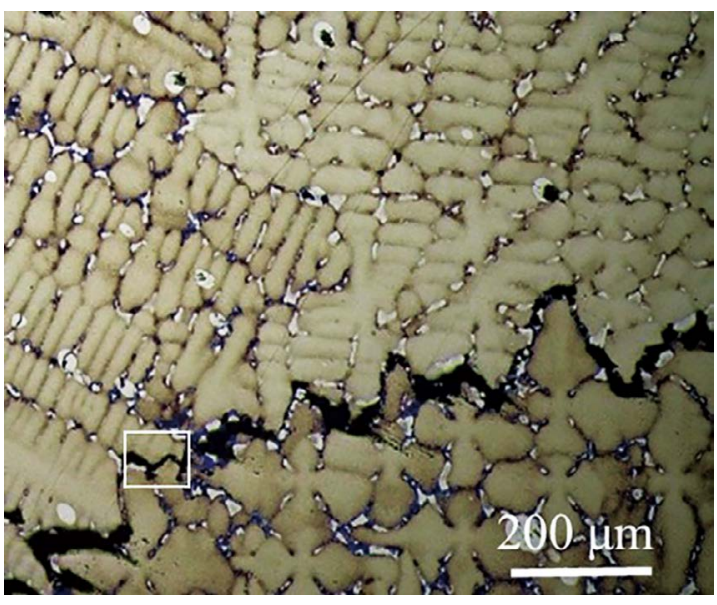

Fig. 7: Magnified microstructure of the zone marked by white square in Fig. 6
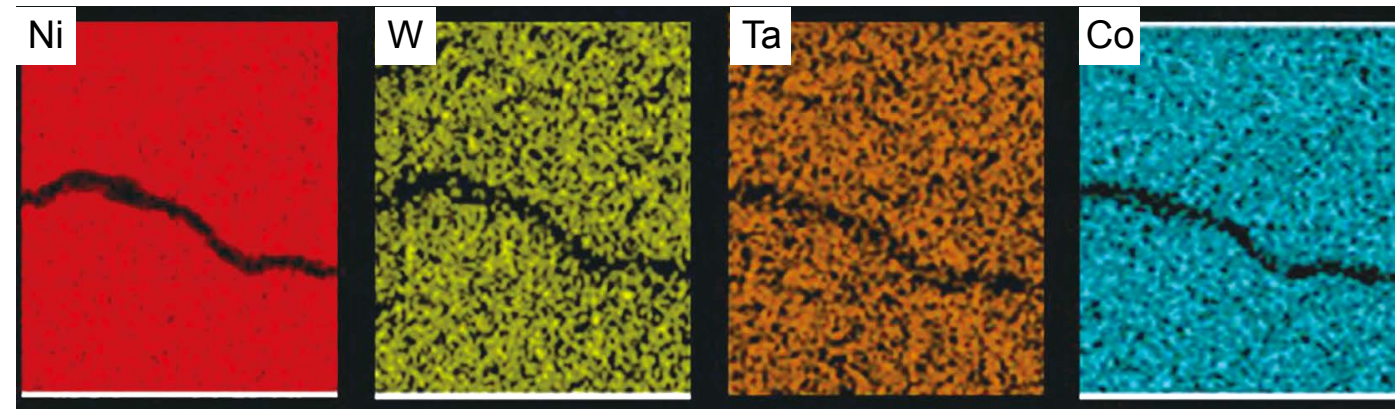

Fig. 8: EDS results of the zone marked by white square in Fig. 7

a hot crack formed at the grain boundary. Since the stray grain region directly contacts the center region, and the center region is marginally occupied, some stray grains gradually enter the center region during the subsequent directional solidification. Therefore, some stray grains appear in the blade body above Platform 3, as shown in Fig. 9.

The microstructure of the bottom surface of Platform 2 of the dummy blade with a misorientation of $15^{\circ}$ is shown in Fig. 10. Similar to Platform 2 of the dummy blade with $<001>$

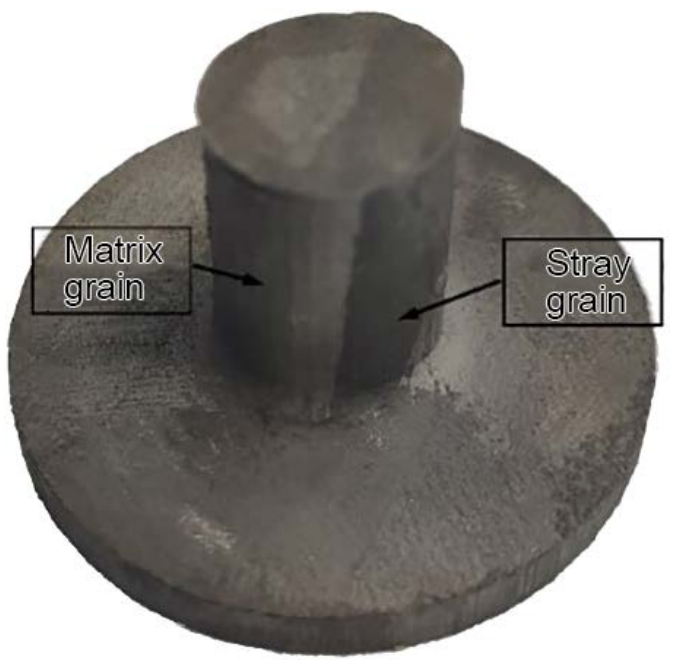

Fig. 9: Macrostructure of Platform 3 and blade body above it orientation, Platform 2 with a misorientation of $15^{\circ}$ was also divided into the center, expansion, and stray grain regions with some new characteristics. The dendrite is arranged in line at the central region, which is obviously different from that of dummy blade with $<001>$ orientation. The dendrite morphology at the expansion region is related with the secondary orientation, which is defined by the $<010>$ direction. Because the $<010>$ direction is parallel to the bottom surface of platform, a long-term secondary dendrite is observed, as shown in Fig. 10(c). Otherwise, as shown in Fig. 10(d), since other direction is tilted to the bottom surface of the platform, a truncated dendrite is observed. The area of the stray grain region is larger than that with $<001>$ orientation.

\section{Discussion}

Nucleation and dendrite deformation are the two major mechanisms in the formation of stray grains during the directional solidification of Ni-based single-crystal superalloys ${ }^{[22]}$. According to a report by Sun et al. ${ }^{[18,19]}$, long secondary dendrite arms can grow throughout a square platform, resulting in a convergent boundary between the corresponding branching dendrite and the expanding secondary arm from the blade body. Their analysis on orientation detection showed that the misorientation between the two sides of the convergent boundary monotonically increased from one platform edge to the other, resulting in a large-angle grain boundary. Therefore, 
(a)

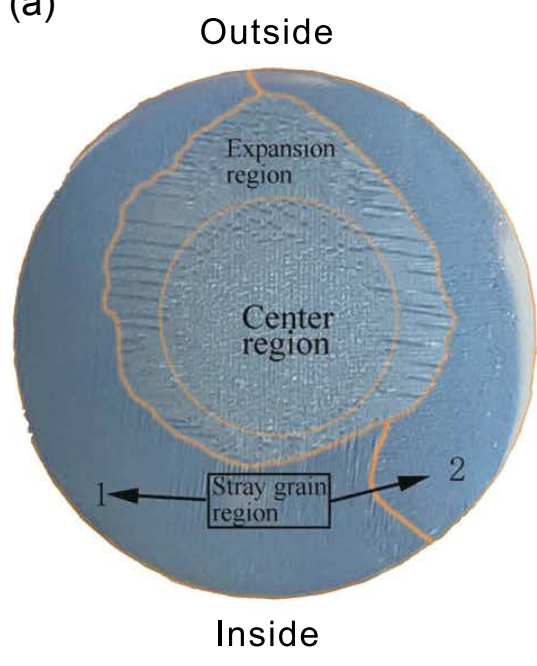

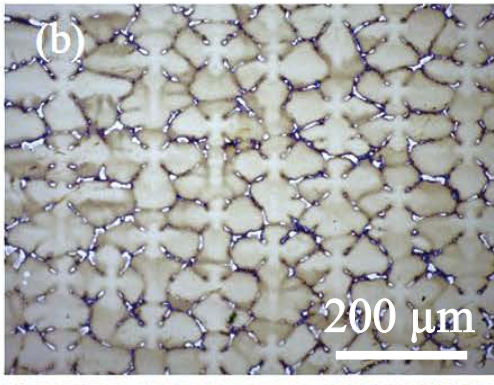
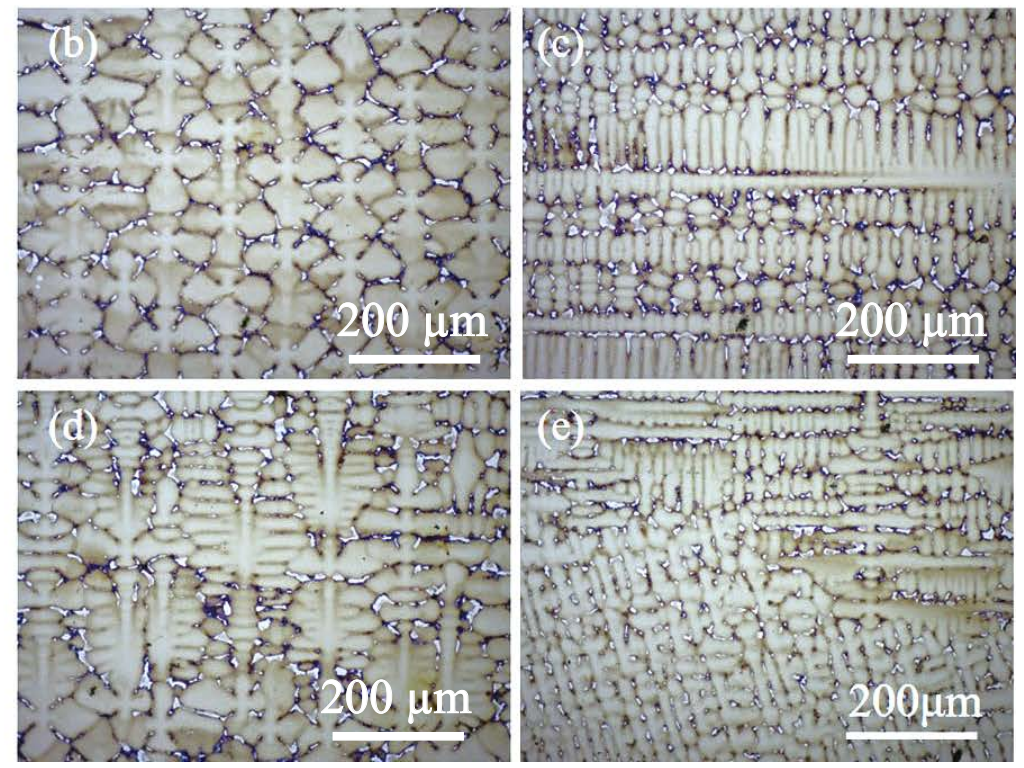

Fig. 10: Transverse macrostructure (a) and microstructure of Platform 2 of dummy blade with a misorientation of $15^{\circ}$ in center region (b), expansion region (c) and (d), and stray grain region (e)

the convergent boundary gradually becomes easier to distinguish. However, the misorientation between the two sides of the grain boundary caused by nucleation remained basically unchanged, and the grain boundary is easily distinguished in all positions on the blade.

The dendrite branching growth behavior on a circular platform is significantly different from that on a square platform, because the edge of a square platform is the first position to reach a temperature below the liquidus isotherm ${ }^{[5,23]}$, and the matrix grain fills this region through high-order branching. Then, as the center region reaches a temperature below the liquidus isotherm, the dendrites at the edge of the platform branch into new dendrites to fill this region. Therefore, a dendritic loop forms on the square platform. However, because a circular platform has no edges aside from a circular outside edge, high-order branching from dendrites in the center region only continues growing to the circular outside edge or meets the dendritic front of the stray grain region without forming a dendrite loop. Combining the above analysis and the characteristics of the grain boundaries shown in Figs. 3-5, it can be inferred that the stray grains formed in the circular platforms are caused by a nucleation mechanism.

The nucleation of stray grains on a platform is closely related to the evolution of the temperature field during the directional solidification process ${ }^{[12,22,24]}$ and the undercoolability of the alloy ${ }^{[17]}$. Li et al. ${ }^{[23]}$ used a combination of experiments and numerical simulations to study the evolution of the temperature field during directional solidification in a dummy blade with a square platform and its effect on the formation of stray grains. They found that the nucleation of stray grains was governed by a competition between the time needed for dendrite branching and cooling, which was affected by the undercoolability, temperature gradient at the solidification front, and withdrawal rate. Because the dummy blade was located at the edge of the mold, the temperature field became asymmetric during directional solidification of the multi-blade cluster, as shown in Fig. 11. According to the numerical simulation results reported by Ma et al. ${ }^{[13]}$, the macroscopic liquidus isotherm is located in the heat zone of the directional solidification furnace and has a convex shape, resulting in a large undercooling degree and undercooling time before the platform is filled by high-order branching from the matrix grain inside a square platform. Therefore, the nucleation of stray grains occur easily inside the platform. At the same time, because the liquidus isotherm is convex toward the heat zone of the directional solidification furnace, the undercooling degree at the outside of a circular platform is small, and thus the probability of stray grain nucleation is also relatively small.

With an increase in the solidification distance, the cooling effect of the water-cooled copper chill plate gradually decreases, and the liquidus isotherm further penetrates the cooling zone of the directional solidification furnace, as shown in Fig. 11(b). Therefore, the liquidus isotherm becomes more convex, and the undercooling degree and undercooling time increase at the inside of the circular platform. At the same time, the temperature reduction in the solidified zone far from the liquidus isotherm causes an increase in the gap value between the casting and mold and a decrease in the heat transfer coefficient between the casting and mold. Therefore, the difference in cooling rate between the casting and mold increases, and the temperature varies greatly between the casting and mold in the mush zone. This further increases the undercooling degree and undercooling time, which allows stray grains to nucleate on the inside of the circular platform. Therefore, the stray grain area increases with the increasing distance between the circular platform and the bottom of the cluster in this experiment, as shown in Fig. 12.

Crystal orientation is one of the important factors affecting the microstructure evolution of a platform. As shown in Fig. 12, 


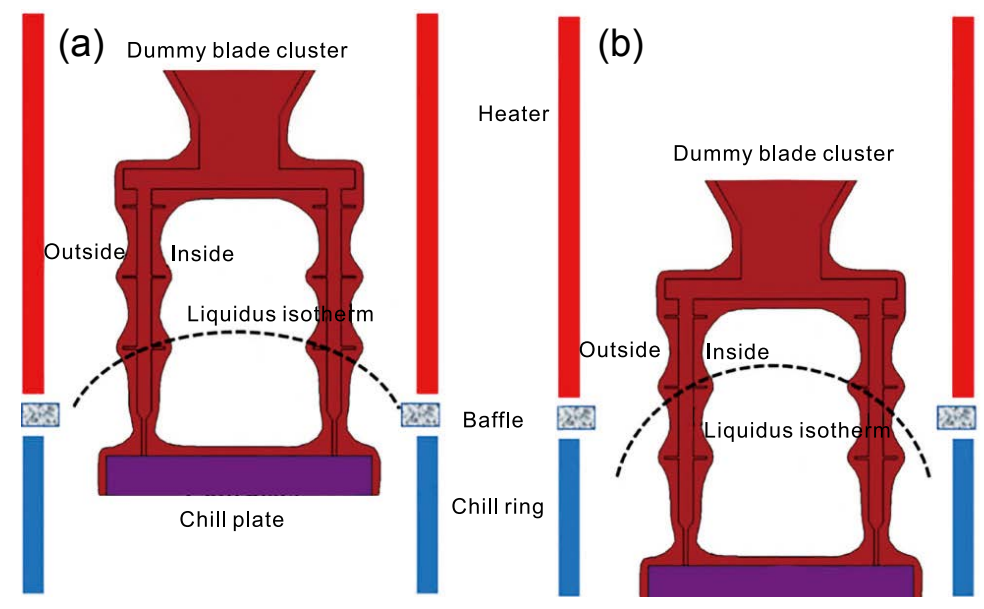

Fig. 11: Schematic of liquidus isotherm development at different heights during the directional solidification of a blade cluster for Platform 1 (a) and Platform 2 (b)
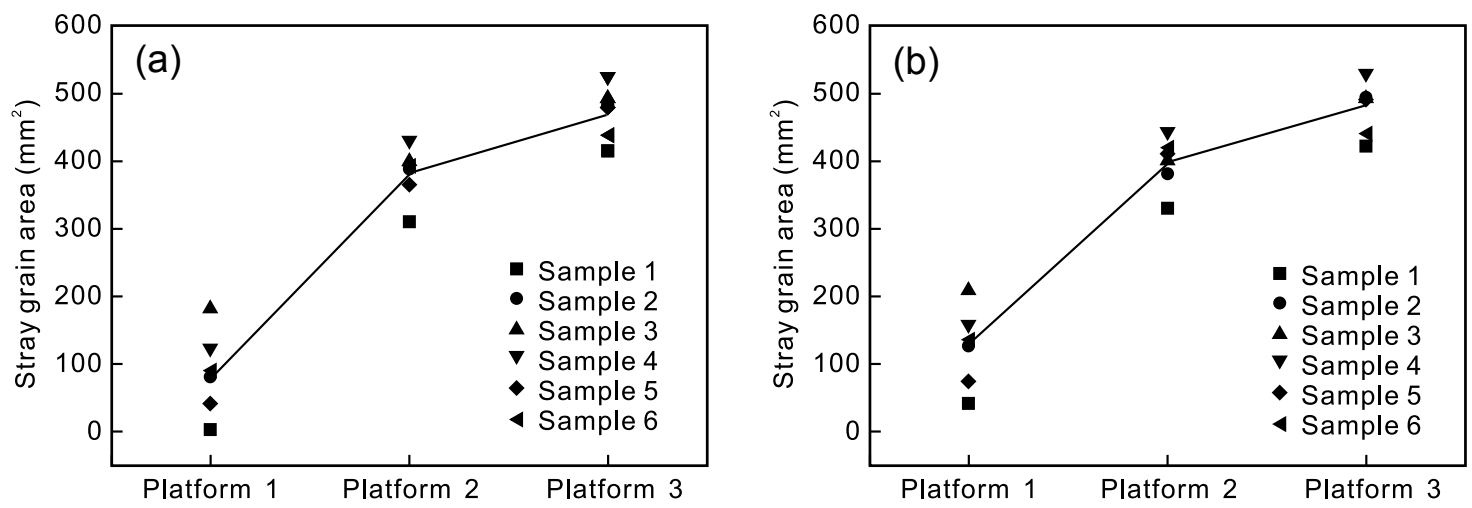

Fig. 12: Stray grain areas on different circular platforms: (a) dummy blade with <001> orientation; (b) dummy blade with misorientation of $15^{\circ}$

the stray grain area slightly increases as the crystal orientation of the dummy blade changes from $<001>$ orientation to a misorientation of $15^{\circ}$. This is because of the difference in dendrite growth behavior caused by the crystal orientation. As shown in Fig. 13(a), long secondary dendrite arms can grow in the entire platform of a dummy blade with $<001>$ orientation. However, when the $<001>$ direction deviates from the dummy blade's axis, dendrites diverge from [Fig. 13(b)] or converge into [Fig. 13(c)] the platform. Continuous high-order branching of dendrite occurs and spreads to the edge of the platform, causing the speed of dendrite branching to the edge of the platform to

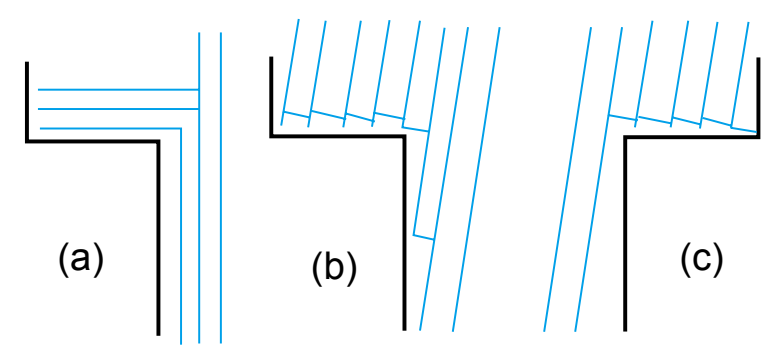

Fig. 13: Schematic of dendrite growth at platform of dummy blade with a misorientation of $15^{\circ}$ :

(a) $<001>$ orientation, (b) diverging and (c) converging platform be lower than that of the $<001>$ orientation. Consequently, the area of the platform expansion is reduced. Notably, the influence of crystal orientation on the formation of stray grains is significantly weaker than the temperature field evolution during directional solidification.

\section{Conclusion}

An experimental study was conducted to investigate the mechanism underlying the influence of the position of a circular platform on the nucleation of stray grains during the preparation of a $<001>$ oriented single-crystal dummy blade cluster. The microstructure of the circular platform consists of a center region, expansion region, and stray grain region. The inside of the circular platform (facing the center of the cluster) is more prone to stray grain nucleation than the outside (facing the furnace body). With an increase in the distance between the circular platform and the bottom of the dummy blade cluster, the stray grain area increases, whereas the expansion area decreases. The stray grain area is slightly aggravated with the increase of the misorientation. A hot crack forms at the grain boundary between the stray grain and center region. The formation of defects is mainly controlled by the temperature field evolution process during directional solidification. 


\section{References}

[1] Reed R C. The superalloys fundamentals and applications. Cambridge University Press, New York, 2006.

[2] Mclean M. Directional solidified materials for high temperature service. The Metals Society, London, 1983.

[3] Caron P, Cornu D, Khan T, et al. Development of a hydrogen resistant superalloy for single crystal blade application in rocket engine turbopumps. Superalloys, 1996: 53-60.

[4] Meng X B, Li J G, Chen Z Q, et al. Effect of platform dimension on the dendrite growth and stray grain formation in a $\mathrm{Ni}$ base single-crystal superalloy. Metallurgical and Materials Transactions A, 2012, 44(4): 1955-1965.

[5] Li Y F, Liu L, Huang T W, et al. Multi-scale characterization of stray grain in the platform of nickel-base single crystal turbine blade. Vacuum, 2016, 131: 181-187.

[6] Xuan W D, Li C T, Zhao D K, et al. Effect of primary dendrite orientation on stray grain formation in cross-section change region during the directional solidification of $\mathrm{Ni}$-based superalloy. Metallurgical and Materials Transactions B, 2017, 48(1): 394-405.

[7] Qiu F, Bu K, Zheng B, et al. Control of edge plate stray grain of single-crystal turbine blade by using process bar method. International Journal of Metalcasting, 2020, 14(1): 144-154.

[8] Xuan W D, Zhang H W, Shao W, et al. Formation Mechanism of stray grain of nickel-based single-crystal superalloy under a high magnetic field during directional solidification. Metallurgical and Materials Transactions B, 2019, 50(1): 27-31.

[9] Rausch A M, Pistor J, Breuning C, et al. New grain formation by constitutional undercooling due to remelting of segregated microstructures during powder bed fusion. Materials, 2021, 14(12): 3324.

[10] Ma D X, Bührig-Polaczek A. Application of a heat conductor technique in the production of single-crystal turbine blades. Metallurgical and Materials Transactions B, 2009, 40(5): 738-748.

[11] Meyer T V M, Dedecke D, Paul U, et al. Undercooling related casting defects in single crystal turbine blades. Superalloys, 1996: 471-479.

[12] Meng X B, Li J G, Zhu S Z, et al. Method of stray grain inhibition in the platforms with different dimensions during directional solidification of a Ni-base superalloy. Metallurgical and Materials Transactions A, 2013, 45(3): 1230-1237.
[13] Ma D X, Wang F, Wu Q, et al. Temperature evolution and grain defect formation during single crystal solidification of a blade cluster. China Foundry, 2017, 14(5): 456-460.

[14] Yang X, Dong H B, Wang W, et al. Microscale simulation of stray grain formation in investment cast turbine blades. Materials Science and Engineering A, 2004, 386(1-2): 129-139.

[15] Gao S F, Liu L, Zhang J, et al. Simulation of stray grain formation at the platform during Ni-base single crystal superalloy DD403 casting. China Foundry, 2015, 12(2): 118-122.

[16] Zhang X L, Zhou Y Z, Jin T, et al. Study on the tendency of stray grain formation of Ni-based single crystal superalloy. Acta Metallurgica Sinica, 2012, 48(10): 79-86.

[17] Wang H W, Ma D X, Yang G X, et al. Study on the undercoolability and single crystal castability of Nickel-based superalloys. In: Liu X, et al. (eds.) Energy Materials 2017, The Minerals, Metals \& Materials Series. Springer, Cham: 295-302.

[18] Sun D J, Liu L, Huang T W, et al. Insight of the dendrite deformation in Ni-based superalloys for increased misorientation along convergent boundaries. Progress in Natural Science Materials International, 2018, 28(4): 489-495.

[19] Sun D J, Liu L, Huang T W, et al. Dendrite growth and orientation evolution in the platform of simplified turbine blade for nickel-based single crystal superalloys. Acta Metallurgica Sinica, 2019, 55(5): 619-626.

[20] Sun D J, Liu L, Yang W T, et al. Influence of secondary dendrite orientation on the evolution of misorientation in the platform region of single crystal superalloy turbine blades. Advanced Engineering Materials, 2019, 21(2): 1800933.

[21] Carney C A, Beech J. The origin of sliver defects in single crystal turbine blades. In: Proc. Solidification Processing. London: Institute of Metals, 1987.

[22] Zhou Y Z. Formation of stray grains during directional solidification of a nickel-based superalloy. Scripta Materialia, 2011, 65(4): 281-284.

[23] Li Y F, Liu L, Sun D J, et al. Quantitative analysis of withdrawal rate on stray grain formation in the platforms of a Ni-Based single crystal dummy blade. Journal of Alloys and Compounds, 2019, 773: 432-442.

[24] Li Y F, Liu L, Huang T W, et al. The process analysis of seeding-grain selection and its effect on stray grain and orientation control. Journal of Alloys and Compounds, 657: 341-347. 